\title{
Resultado visual de la cirugía de catarata en niños del Hospital San Felipe Enero 2001-Enero 2017
}

Visual results of cataract surgery in children at Hospital san Felipe January 2001-january 2017

\section{Fabiola Jerez Carvajal*, Alberto Ehrler**, Iván Espinoza**}

\section{RESUMEN}

Antecedentes: La incidencia de catarata en niños es 1/6-10 000 nacimientos. El uso de lente intraocular (LIO) es controversial. Objetivo: Determinar el método de corrección de agudeza visual (AV) más efectivo utilizado en pacientes pediátricos operados de catarata en la Consulta Externa de Oftalmología del Hospital San Felipe de enero 2001enero 2017. Pacientes y métodos: Se realizó un estudio observacional cuantitativo, descriptivo, transversal y retrospectivo en la Consulta Externa de Oftalmología del Hospital San Felipe, Hospital de Referencia Nacional. El universo y la muestra correspondió a 90 ojos de 71 pacientes pediátricos con diagnóstico de catarata en la consulta externa de oftalmología del Hospital San Felipe durante al periodo enero 2001-enero 2017. Se incluyó todo ojo de paciente de 6 meses a 14 años de edad operado de catarata en Hospital San Felipe. Se excluyó todo ojo de paciente pediátrico con diagnóstico de catarata que tuviese otra condición que comprometiera su AV (desprendimiento de retina, cicatriz macular, atrofia óptica, leucoma corneal) y/o que no hubiese sido intervenido quirúrgicamente por la catarata. Resultados: Se encontraron 90 ojos operados: $40.45 \%$ cataratas congénitas y $35.96 \%$ traumáticas. La agudeza visual previa de cuenta

\footnotetext{
*Médico residente de tercer año de Oftalmología Pediátrica, Instituto Nacional de Pediatría.

** Médico Oftalmólogo subespecialista en Oftalmología Pediátrica y Estrabismo, UNAH.

***Unidad de Investigación Científica, Facultad de Ciencias Médicas, UNAH

Dirigir correspondencia a: fabijerez.c@gmail.com

Recibido: 20 de Enero $2019 \quad$ Aprobado: 15 de Agosto 2019
}

dedos - percepción de luz (CD-PL) en un $71.1 \%$ de los casos. Al $47.8 \%$ de los casos se practicó EEC+LIO CP. Complicaciones transoperatorias: ruptura de cápsula posterior $(15.5 \%)$ y 1 luxación de cristalino a cámara vítrea. La agudeza visual obtenida posterior a la cirugía entre $20 / 20-20 / 40$ se obtuvo con lente intraocular (52.4\%). Conclusiones: La mejor AV se obtuvo con LIO.

\section{PALABRAS CLAVE}

Catarata Pediátrica, Agudeza Visual Postquirurgica, Pseudofaquia Infantil.

\section{ABSTRACT \\ Introduction: The incidence of cataract in children is $1 / 6-10000$ births. The use of intraocular (IOL) lens is still controversial. Objective: Determine the most effective method of visual acuity (VA) correction in pediatric patients with cataract surgery in Hospital San Felipe from January 2001-January 2017. Material and} methods: A quantitative, descriptive, transverse and retrospective observational study was carried out at the Ophthalmology Outpatient Clinic of the Hospital San Felipe, National Reference Hospital. The universe and the sample corresponded to 90 eyes of 71 pediatric patients with a diagnosis of cataracts who attended the outpatient clinic of ophthalmology of the Hospital San Felipe during the period January 2001 to January 2017 . The study included all eyes of pediatric patients from 6 months to 14 years of age who had cataract surgery at Hospital San Felipe. All eyes of pediatric patients with a diagnosis of cataract that had another 
condition that compromised their VA (retinal detachment, macular scarring, optic atrophy, corneal leukoma) and / or had not been surgically treated by cataract were excluded. The previous visual acuity was finger countlight perception (FC-LP) in $71.11 \%$ of the cases, $47.78 \%$ of the cases had EEC + IOL $\mathrm{CP}$. Within the transurgical complications posterior capsule rupture was most common $82.35 \%$ out of $18.89 \%$, there was also 1 lens luxation to the vitreous chamber. Visual acuity after surgery of 20/20 -20/40 was acomplished with Intraocular Lens (IOL) $52.4 \%$ of the cases. Conclusions: The best corrected Visual Acuity was obtained with IOL.

\section{KEY WORDS}

Pediatric Cataract, Postsurgical Visual Acuity, pseudofaquia in children.

\section{INTRODUCCIÓN}

La incidencia de catarata en niños es menor que en adultos, siendo 1/6-10 000 nacimientos(1). Los pacientes con catarata monocular tienen 2 factores predisponentes para desarrollar ambliopía: rivalidad binocular y deprivación visual. Para prevenirlo los pacientes con cataratas densas deben ser operados antes de las 17 semanas. El uso de lente intraocular aún es controversial ya que entra en juego la edad mínima para implantar el lente factores predisponentes para desarrollar ambliopía: rivalidad binocular y deprivación visual. Para prevenirlo los pacientes con cataratas densas deben ser operados antes de las 17 semanas. El uso de lente intraocular aún es controversial ya que entra en juego la edad mínima para implantar el lente intraocular, la selección del poder refractivo del mismo, opacidad de cápsula posterior consecuente, complicaciones a largo plazo como el glaucoma y el desprendimiento regmatógeno de retina. ${ }^{(1,2)}$ En niños menores de 2 años puede haber un colapso escleral intraoperatorio, presión vítrea, elasticidad aumentada de capsula anterior y posterior, miosis, liberación de fibrina. ${ }^{(2)}$ El tratamiento quirúrgico de cataratas pediátricas con lentes intraoculares es un gran reto debido a la rigidez escleral, elasticidad de la cápsula anterior y el tamaño pequeño del ojo. En ojos pediátricos normales la longitud axial aumenta rápidamente entre los 2-3 años de edad, decrece y se estabiliza entre los 8-10 años de edad. Al contrario, la curvatura corneal disminuye con la edad y se estabiliza al año de edad.(3) La edad para implante un lente intraocular (LIO) versus dejar el niño en afaquia ha sido tema de debate por muchos años. Según Lambert los niños mayores de 6 meses de edad tienen un mejor resultado visual que aquellos que se corrigen con lentes de contacto. El estudio de Tratamiento de Afaquia Infantil (IATS) encontró que al año de tratamiento con LIO versus Lente de contacto no hubo una diferencia significativa en la agudeza visual de los pacientes.

Sin embargo, las complicaciones fueron mayores en el grupo de LIO con pacientes que se tuvieron que reintervenir después del implante primario. ${ }^{(3,4)}$ La complicación postoperatoria más frecuente es la opacidad de cápsula posterior en un $95 \% .{ }^{(5)}$ La cirugía de catarata con LIO puede proveer de beneficios en el resultado visual final en niños que son intervenidos antes de que se desarrolle una función foveolar anormal. ${ }^{(6,7)} \mathrm{La}$ corrección afaquica se ha alcanzado tradicionalmente con el uso de gafas y/o lentes de contacto. Las gafas están asociadas a aberraciones y no se utilizan mucho por razones cosméticas. El uso de lentes de contacto requiere de colaboración del paciente y de los padres. ${ }^{(7,8)}$ La escasa caracterización de la catarata pediátrica llevó a plantear el siguiente estudio que iba dirigido a determinar el método de corrección de agudeza 
visual más efectivo utilizado en los pacientes pediátricos operados de catarata en la Consulta Externa de Oftalmología del Hospital San Felipe para dar a conocer el resultado de agudeza visual de dichos pacientes.

\section{PACIENTES Y MÉTODOS}

Este estudio fue realizado en la Consulta Externa de Oftalmología del Hospital San Felipe, Hospital de Referencia Nacional, mediante un diseño observacional cuantitativo, descriptivo, transversal y retrospectivo. Se realizó una revisión de 102 expedientes encontrados con diagnóstico de catarata pediátrica de los cuales el universo y la muestra correspondió a 90 ojos de 71 pacientes pediátricos que acudieron a la consulta externa de oftalmología del Hospital San Felipe durante al periodo enero 2001- enero 2017.

Dentro de los criterios de inclusión se tomó en cuenta a todo paciente pediátrico comprendido a las edades ya descritas en el periodo de tiempo establecido. Dentro de los criterios de exclusión se omitió todo paciente pediátrico con diagnóstico de catarata que tuviese otra condición que comprometiera su agudeza visual (desprendimiento de retina, cicatriz macular, atrofia óptica, leucoma corneal) y/o que no hubiese sido intervenido quirúrgicamente por la catarata. Se utilizó como fuente primaria de datos todos los expedientes de los pacientes que fueron operados de catarata en el Servicio Oftalmología antes descrito. Se llenó un instrumento de preguntas cerradas conteniendo entre las variables más relevantes: agudeza visual, técnicas de corrección de afaquia, características sociodemográficas, complicaciones quirúrgicas y técnicas quirúrgicas. La información recolectada fue ingresada en una base de datos que se creó usando el paquete estadístico EPIINFO Ver- sión 7.0. 9.34 (CDC, Atlanta EUA). Se realizó una limpieza de las bases de datos para revisar inconsistencias, valores fuera de rango y otros errores de digitación o de colección de datos. Se creó un diccionario de datos y el análisis básico consistió en construcción de frecuencias simples de las variables y análisis univariado y bivariado; se aplicó Chi cuadrado para comparación de proporciones y t-student para comparación de medias en los casos que fueron necesarios. El estudio fue sometido a dictamen del Comité de Ética en Investigación Biomédica de la FCM/UNAH así también se obtuvo la autorización de la Jefatura del Departamento de Oftalmología, y de la Dirección del Hospital San Felipe. Entre las dificultades metodológicas se resaltan la falta de expedientes, la pérdida de información del año 2000 y 2007, la variabilidad de la información consignada en las evaluaciones posteriores a la cirugía, datos incompletos ó no consignados.

\section{RESULTADOS}

Según el departamento de estadística del Hospital San Felipe, se encontraron 189 pacientes diagnosticados con catarata pediátrica entre enero 2001-enero 2017 de los cuales se encontraron 102 expedientes.

En la revisión de estos se encontró una muestra de 90 ojos de 71 pacientes aptos para este estudio. Dentro de las características sociodemográficas la edad más frecuente en la que se realizó la cirugía de catarata corresponde a un rango de 6 años-10 años (63.33\%), el $33.33 \%$ de los niños cursan de 4to-6to grado, no hay predominio marcado entre género: el sexo femenino $50.56 \%$ y masculino $49.44 \%$ respectivamente. La mayoría de los pacientes proceden del departamento de Francisco Morazán (35.56\%). (Ver Cuadro No. 1). 
Cuadro No. 1: Cirugía de catarata pediátrica de 6 meses - 14 años de edad del hospital san Felipe enero 2001 - enero 2017 / $(n=90)$.

\begin{tabular}{llc}
$\begin{array}{l}\text { DATOS SOCIO- } \\
\text { DEMOGRÁFICOS }\end{array}$ & $\mathbf{N}$ & $\%$ \\
\hline EDAD & & \\
$11 \mathrm{a}-14 \mathrm{a}$ & 11 & $12.22 \%$ \\
$6 \mathrm{a}-10 \mathrm{a}$ & 57 & $63.33 \%$ \\
$6 \mathrm{~m}-5 \mathrm{a}$ & 22 & $24.44 \%$ \\
Total & 90 & $100.00 \%$
\end{tabular}

\section{ESCOLARIDAD}

4TO-6TO GRADO

$30 \quad 33.33 \%$

KINDER - 3ER GRADO

NINGUNA

$10.00 \%$

NO CONSIGNADO

SECUNDARIA

Total

PROCEDENCIA

$\begin{array}{lcl}\text { CHOLUTECA } & 7 & 7.8 \% \\ \text { COMAYAGUA } & 12 & 13.3 \% \\ \text { CORTES } & 4 & 4.4 \% \\ \text { EL PARAISO } & 10 & 11.1 \% \\ \text { FCO. MORAZAN } & 32 & 35.6 \% \\ \text { INTIBUCA } & 2 & 2.2 \% \\ \text { LA PAZ } & 5 & 5.6 \% \\ \text { NO CONSIGNADO } & 3 & 3.3 \% \\ \text { OLANCHO } & 8 & 8.9 \% \\ \text { SANTA BARBARA } & 2 & 2.2 \% \\ \text { VALLE } & 4 & 4.4 \% \\ \text { YORO } & 1 & 1.1 \% \\ \text { Total } & 90 & 100.00 \%\end{array}$

SEXO

\begin{tabular}{lll} 
FEMENINO & 45 & $50.0 \%$ \\
MASCULINO & 45 & $50.0 \%$ \\
\hline Total & 90 & $100.0 \%$
\end{tabular}

Fuente: Resultado visual de la cirugía de catarata en niños del Hospital San Felipe Enero 2001-Enero 2017
El $40.45 \%$ de las cataratas diagnosticadas en el servicio de oftalmología pediátrica del Hospital San Felipe son congénitas seguidas de las cataratas traumáticas que corresponden a un $35.96 \%$. (Ver Cuadro 2).

Cuadro No. 2: Tipo de catarata pediátrica diagnosticadas en niños de 6 meses - 14 años de edad del hospital san Felipe enero 2001 - enero 2017 ( $n=90)$.

\begin{tabular}{lcc}
\hline DIAGNÓSTICO & $\mathbf{N}$ & $\%$ \\
\hline CATARATA & & \\
CONGENITA & 36 & $40.00 \%$
\end{tabular}

CATARATA POLAR

$\begin{array}{lll}\text { POSTERIOR } & 9 & 10.00 \%\end{array}$

CATARATA REDUCTA $1 \quad 1.11 \%$

CATARATA

$\begin{array}{lll}\text { TRAUMATICA } & 32 & 35.56 \%\end{array}$

CATARATA UVEÍTICA $22.22 \%$

NO CONSIGNADO $\quad 7 \quad 7.78 \%$

$\begin{array}{lll}\text { OTRO } & 3 & 3.33 \%\end{array}$

Total $\quad 90 \quad 100 \%$

Fuente: Resultado visual de la cirugía de catarata en niños del Hospital San Felipe Enero 2001-Enero 2017

Se descubrió que la agudeza visual del ojo afectado previo a la intervención quirúrgica es baja, correspondiendo el $71.11 \%$ a un rango de Cuenta Dedos - Percepción de Luz (CD-PL). (Ver Cuadro 3). 
Cuadro No. 3: Resultado agudeza visual previa a la cirugía de catarata en niños del hospital san Felipe $(n=90)$.

\begin{tabular}{lcc}
\hline AVPREVIO & N & \multicolumn{1}{c}{$\%$} \\
\hline $20 / 100-20 / 400$ & 6 & $6.67 \%$ \\
$20 / 50-20 / 80$ & 3 & $3.33 \%$ \\
CD-PL & 64 & $71.11 \%$ \\
CSM & 8 & $8.89 \%$ \\
NO CONSIGNADO & 9 & $10.00 \%$ \\
\hline Total & 90 & $100 \%$
\end{tabular}

Fuente: Resultado visual de la cirugía de catarata en niños del Hospital San Felipe Enero 2001-Enero 2017

La técnica quirúrgica más utilizada más frecuente fue la extracción extracapsular de catarata más colocación de Lente Intraocular en Cápsula Posterior en un $47.78 \%$ ( Ver cuadro 4).

Cuadro No. 4: Técnica quirúrgica utilizada en la cirugía de catarata pediátrica de 6 meses- 14 años de edad del hospital san Felipe enero 2001- enero 2017 ( $n=90)$.

\begin{tabular}{lcc}
\hline TECNICAQX & N & $\%$ \\
\hline EEC+IP & 36 & $40.00 \%$ \\
EEC+LIO CP & $\mathbf{4 3}$ & $\mathbf{4 7 . 7 8 \%}$ \\
MICS+IP & 2 & $2.22 \%$ \\
MICS+LIO CP & 5 & $5.56 \%$ \\
OTRO & 4 & $4.44 \%$ \\
\hline Total & 90 & $100 \%$
\end{tabular}

Fuente: Resultado visual de la cirugía de catarata en niños del Hospital San Felipe Enero 2001-Enero 2017
Durante las cirugías efectuadas el $18.89 \%$ tuvo complicaciones (Ver Cuadro 5), la mayoría ruptura de Cápsula posterior $82.35 \%$ y una luxación de cristalino a cámara Vítrea conformando el 5.88\%.

Cuadro No. 5: Complicaciones durante la cirugía de catarata en niños de 6 meses14 años de edad del hospital san Felipe enero 2001- enero 2017 ( $n=17)$.

\begin{tabular}{lcr}
\hline COMPLICACIONES & N & \multicolumn{1}{c}{$\%$} \\
\hline DC & 0 & $00.00 \%$ \\
DR & 0 & $00.00 \%$ \\
LUXACIONDELIOACV & 1 & $5.88 \%$ \\
RUPTURACP & 14 & $82.35 \%$ \\
OTROS & 2 & $11.77 \%$ \\
\hline Total & 17 & $100 \%$
\end{tabular}

Fuente: Resultado visual de la cirugía de catarata en niños del Hospital San Felipe Enero 2001-Enero 2017

La mayoría de los pacientes intervenidos se les colocó lente intraocular (55.6\%), seguido de lentes aéreos $(28.9 \%)$, lentes de contacto $(8.9 \%)$. (Ver cuadro 6$)$.

La mayoría de pacientes contaba con una mala agudeza visual previo a la cirugía que se encontraba en un rango de Cuenta Dedos - Percepción de Luz (CD-PL) en el $71.1 \%$ de los casos. Sin embargo, su agudeza visual posterior a la cirugía mejoró en su mayoría a intraocular (38.9\%) seguido de corrección con lente de contacto y lente aéreo ambos en $22.2 \%$. 
Cuadro No. 6: Resultado visual posterior de la cirugía de catarata en niños de 6 meses14 años de edad del hospital san Felipe enero 2001- enero 2017 ( $n=90)$.

\section{CORRECCIÓN DE AFAQUIA CON:}

\begin{tabular}{|c|c|c|c|c|c|}
\hline $\begin{array}{c}\text { AGUDEZA } \\
\text { VISUAL } \\
\text { POSTERIOR } \\
\text { A LA } \\
\text { CIRUGIA } \\
\end{array}$ & $\begin{array}{l}\text { LENTE } \\
\text { AÉREO } \\
\mathrm{N}(\%)\end{array}$ & $\begin{array}{l}\text { LENTE DE } \\
\text { CONTACTO }\end{array}$ & $\begin{array}{l}\text { LENTE } \\
\text { INTRAO- } \\
\text { CULAR }\end{array}$ & $\begin{array}{c}\text { NO } \\
\text { CONSIGNADO }\end{array}$ & $\begin{array}{c}\text { Total N } \\
(\%)\end{array}$ \\
\hline $20 / 20-20 / 40$ & $10(47.6 \%)$ & $0(0.0 \%)$ & $11(52.4 \%)$ & $0(0.0 \%)$ & $\begin{array}{l}21 \\
(100.0 \%) \\
18\end{array}$ \\
\hline $20 / 50-20 / 80$ & 2 (11.1\%) & $0(0.0 \%)$ & $15(83.3 \%)$ & $1(5.6 \%)$ & $\begin{array}{l}(100.0 \%) \\
14\end{array}$ \\
\hline $20 / 100-20 / 400$ & $4 \quad(28.6 \%)$ & $4(28.6 \%)$ & $6 \quad(42.9 \%)$ & $0(0.0 \%)$ & $\begin{array}{l}(100.0 \%) \\
18\end{array}$ \\
\hline CD-PL & $4 \quad(22.2 \%)$ & $4(22.2 \%)$ & $7 \quad(38.9 \%)$ & $3(16.7 \%)$ & $\begin{array}{l}(100.0 \%) \\
2\end{array}$ \\
\hline \multirow{2}{*}{$\begin{array}{l}\text { CSM } \\
\text { NO } \\
\text { CONSIGNADO }\end{array}$} & $2(100.0 \%)$ & $0(0.0 \%)$ & $0 \quad(0.0 \%)$ & $0(0.0 \%)$ & $(100.0 \%)$ \\
\hline & $4 \quad(23.5 \%)$ & $0(0.0 \%)$ & $11(64.7 \%)$ & $2(11.8 \%)$ & $\begin{array}{l}17 \\
(100.0 \%)\end{array}$ \\
\hline TOTAL & $26(28.9 \%)$ & $8(8.9 \%)$ & $50(55.6 \%)$ & $6(6.7 \%)$ & $\begin{array}{l}90 \\
(100.0 \%)\end{array}$ \\
\hline
\end{tabular}

Fuente: Resultado visual de la cirugía de catarata en niños del Hospital San Felipe Enero 2001-Enero 2017

Los pacientes obtuvieron agudeza visual de cuenta dedos - percepción de luz con lente 20/20-20/40 en el $23.3 \%$ de los casos seguido de 20/50-20/80 en el 20\%. (Ver Cuadro 7 ).

Cuadro No. 7: Resultado visual previa y posterior de la cirugía de catarata en niños de 6 meses- 14 años de edad del hospital san Felipe enero 2001- enero 2017 ( $n=90)$.

\begin{tabular}{llllll}
\hline AV Previa & N & $\%$ & AV Posterior & N & $\%$ \\
\hline $20 / 20-20 / 40$ & 0 & $0.0 \%$ & $20 / 20-20 / 40$ & 21 & $23.3 \%$ \\
$20 / 50-20 / 80$ & 3 & $3.3 \%$ & $20 / 50-20 / 80$ & 18 & $20.0 \%$ \\
$20 / 100-20 / 400$ & 6 & $6.7 \%$ & $20 / 100-20 / 400$ & 14 & $15.6 \%$ \\
CD-PL & 64 & $71.1 \%$ & CD-PL & 18 & $20.0 \%$ \\
CSM & 8 & $8.9 \%$ & CSM & 2 & $2.2 \%$ \\
NO & & & NO & 17 & $18.9 \%$ \\
CONSIGNADO & 9 & $10.0 \%$ & CONSIGNADO & & \\
\hline Total & 90 & $100.0 \%$ & Total & 90 & $100.0 \%$
\end{tabular}

Fuente: Resultado visual de la cirugía de catarata en niños del Hospital San Felipe Enero 2001-Enero 2017 
Se encontró que el rango de edad más frecuente en el que se realizó la cirugía de catarata fue entre 6-10 años. (Ver cuadro 8).

Cuadro No. 8: Resultado edad y corrección de la cirugía de catarata en niños de 6 meses- 14 años de edad del hospital san felipe enero 2001- enero 2017 ( $n=90)$.

\begin{tabular}{|c|c|c|c|c|c|}
\hline EDAD & $\begin{array}{l}\text { LENTE } \\
\text { AÉREO }\end{array}$ & $\begin{array}{l}\text { LENTE DE } \\
\text { CONTACTO }\end{array}$ & $\begin{array}{c}\text { LENTE } \\
\text { INTRAOCULAR }\end{array}$ & $\begin{array}{c}\text { NO } \\
\text { CONSIGNADO }\end{array}$ & Total \\
\hline $\begin{array}{l}11 a- \\
14 a\end{array}$ & $1(9.1 \%)$ & $0(0.0 \%)$ & $8(72.7 \%)$ & $2(18.2 \%)$ & $\begin{array}{l}11 \\
(100.0 \%)\end{array}$ \\
\hline $\begin{array}{l}6 a- \\
10 a\end{array}$ & $15(26.3 \%)$ & $5(8.8 \%)$ & $34(59.7 \%)$ & $3(5.3 \%)$ & $\begin{array}{l}57 \\
(100.0 \%) \\
22\end{array}$ \\
\hline $6 m-5 a$ & $10(45.5 \%)$ & $3(13.6 \%)$ & $8(36.4 \%)$ & $1(4.6 \%)$ & $(100.0 \%)$ \\
\hline OTA & $26(28.9 \%)$ & $8(8.9 \%)$ & $50(55.6 \%)$ & $6(6.7 \%)$ & $\begin{array}{l}90 \\
(100.0 \%)\end{array}$ \\
\hline
\end{tabular}

Fuente: Resultado visual de la cirugía de catarata en niños del Hospital San Felipe Enero 2001-Enero 2017

\section{DISCUSIÓN}

La incidencia de catarata en niños es menor que en adultos, siento 1/6- 10000 nacimientos. ${ }^{(1)}$ En el postoperatorios se puede presentar uveítis, opacificación de capsula posterior, formación de membranas secundarias, ambliopía. Los problemas intraoperatorios con el riesgo de inflamación postoperatorio, riesgo de ambliopía hacen que la cirugía de catarata en niños sea más compleja e influyen en el resultado visual final.(2) El cálculo promedio del poder lente disminuye 20 dioptrías desde el nacimiento hasta la adultez manteniendo la refracción cercana a emetropía.(3)

El estudio de Tratamiento de Afaquia Infantil (IATS) encontró que al año de tratamiento con LIO versus Lente de contacto no hubo una diferencia significativa en la agudeza visual de los pacientes. Sin embargo, las complicaciones fueron mayores en el grupo de LIO con pacientes que se tuvieron que re-intervenir después del implante primario. ${ }^{(3,4)} \mathrm{Al}$ contrario en este estudio los resul- tados de agudeza visual son muy similares entre pacientes afacos corregidos con lentes aéreos y pacientes con lente intraocular.

La mejor agudeza visual obtenida fue con lente intraocular $(52.4 \%)$ y Lentes aéreos $(47.6 \%)$ logrando una agudeza visual entre 20/20-20/40. El 50\% de los pacientes que utilizaron Lente de contacto alcanzaron agudeza visual entre 20/100-20/400 distinto a lo que expresan los estudios mencionados. La corrección afaquica se ha alcanzado tradicionalmente con el uso de gafas y / o lentes de contacto. Las gafas están asociadas a aberraciones y no se utilizan mucho por razones cosméticas. El uso de lentes de contacto requiere de colaboración del paciente y de los padres. ${ }^{(7,8)}$ Según Morales $M$, en su publicación sobre catarata pediátrica menciona que no se debe implantar LIO en niños menores de 6meses, cosa que tampoco se practica en nuestro hospital. ${ }^{(9)}$

Dentro de las complicaciones que podemos encontrar, la opacificación de la cápsula 
posterior es una de las más frecuentes, teniendo un riesgo del $95 \%$ y esto previene la rehabilitación visual. La incidencia está relacionada con la edad del paciente y con el grado de inflamación postoperatoria que presente. ${ }^{(5)}$ Dentro de las 17 (18.89\%) complicaciones encontradas en este estudio el $82.35 \%$ tuvo ruptura de cápsula posterior y $1(5.88 \%)$ luxación de cristalino a cámara vítrea quien con su corrección refractiva alcanzó una agudeza visual de $20 / 30$. Solo se tomaron en cuenta las complicaciones quirúrgicas para este estudio.

El desarrollo visual tiene un período crítico, durante el cual cualquier insulto que provoque una alteración del estímulo visual normal (uni o bilateral), tendrá como resultado el aparecimiento de ambliopía. El período más sensible se presenta en los primeros 3 años de vida. Zimmerman M y colaboradores mencionan en un estudio sobre la catarata pedíatrica en un país en vías de desarrollo que el propósito principal del diagnóstico precoz de la catarata es evitar las complicaciones irreversibles, principalmente la ambliopía, que influye directamente en el pronóstico visual del niño. ${ }^{(9,10)}$ Aunque la patología congénita ocupa el lugar preponderante en la morbilidad ocular, las lesiones traumáticas en niños en los primeros años de vida, constituyen un factor significativo de daño visual adquirido. La catarata traumática se puede presentar como consecuencia de cualquier tipo de traumatismo ocular tanto contuso como penetrante. Castro et Al menciona en un estudio realizado en servicio de Oftalmología Pediátrica del Instituto Cubano de Oftalmología "Ramón Pando Ferrer" donde se estudiaron 46 pacientes con edades entre los 3 y 14 años entre 2006-2009 con diagnóstico de catarata traumática y agudeza visual mejor corregida menor de 0,4 encontrándose en su mayoría trauma contuso unido al sexo masculino. La relación entre el sexo masculino y femenino en la mayoría de los estudios es de aproximadamente 4:1.(11) Al igual que este estudio la principal causa de catarata pediátrica en Honduras es la congénita $(40.45 \%)$ seguida de la ocasionada por trauma (35.96\%). La edad más frecuente de intervención en este estudio fue entre 6-10 años, lo cual no es lo más apropiado según la literatura mencionada ya que a los 3 años de edad el riesgo de ambliopía es alto.

Ochoa-Gómez dentro de su estudio sobre el manejo quirúrgico y complicaciones de la catarata congénita de cinco años en la Asociación para Evitar la Ceguera en México menciona que la técnica preferida para el manejo de estos casos fue la facoaspiración con colocación de lente intraocular. El mejor manejo de la cápsula posterior para evitar su opacidad fue la facoaspiración con capsulorrexis circular continua posterior y vitrectomía anterior, disminuyendo la opacidad a 9.3\%. La complicación más frecuente fue la opacidad de la cápsula posterior, seguida de la adherencia pupilar (30.4\%) y la subluxación de lente intraocular (2.7\%). ${ }^{(12)}$ Al contrario en este estudio la técnica quirúrgica más utilizada fue la extracción extracapsular más colocación de LIO en capsula posterior (47.78\%) Similar a este estudio en la mayoría de las cirugías se colocó lente intraocular, sin embargo de las complicaciones intraoperatorias más frecuentes se encontró ruptura de cápsula posterior (82.35\%), Luxación de LIO a CV (5.88\%).

Las complicaciones mencionadas en el estudio de Ochoa-Gómez et Al pueden presentarse en diferentes tiempos postquirúrgicos, en este estudio solo se encuentran las complicaciones intraoperatorias. Martínez et al menciona en su estudio sobre cataratas congénitas complicaciones y resultados funcionales que obtuvo mejores resulta- 
dos funcionales cuando implanto LIO primario de forma precoz El $17 \%$ de las cataratas unilaterales y el $23,8 \%$ de las bilaterales consiguieron AV de 0,4 o mejor. ${ }^{(13)}$ La complicación más importante fue la opacificación del eje visual (OEV) que ocurrió en 27 de los 51 ojos. El $50 \%$ de los ojos con LIO primaria y el $63 \%$ de los ojos afáquicos desarrollaron OEV. ${ }^{(14)}$

\section{CONCLUSIONES}

El método de corrección de afaquia con la que se obtuvo la mejor agudeza visual fue muy similiar con Lente intraocular y con lentes aéreos. Hay una mejoría visual notable postquirúrgica, la agudeza visual previa de los pacientes oscila entre CD- PL en su de los pacientes oscila entre CD- PL en su mayoría y la agudeza visual final 20/20-20/40 con lentes intraoculares y lentes aéreos. La catarata congénita es la más frecuente aunque muy similar en frecuencia con la catarata traumática. La técnica quirúrgica más utilizada es la EEC + LIO CP. La mayoría de los niños procedían de Francisco Morazán. 


\section{REFERENCIAS BIBLIOGRÁFICAS}

1. Dorothy SP Fan et Col. Updates on the Surgical Management of Paediatric Cataract with Primary Intraocular Lens Implantation. Ann Acad Med Singapor 2006;35(8):564-70.

2. Ram J, Sukhija J. Pediatric Cataract Management. AIOS, CME series (26) 3. All India Ophthalmological Society 2012.

Hrestha UD. Cataract surgery in children. Nepal J Ophthalmol 2012; 4 (7):138-149.

4. Abhay R. Vasavada and Bharti R. Nihalani. Pediatric cataract surgery. Curr Opin Ophthalmol 2006 17:54-61.

5. Lesueur L. Arné J. Chapatot E. Thouvenin D. Malecaze F. Visual outcome after paediatric cataract surgery: is age a major factor?Br J Ophthalmol 1998; 82:1022-1025.

6. Ondraaek O., Lokaj M. Visual Outcome After Congenital Cataract Surgery. Long-Term Clinical Results Scripta Medica (Brno) 2003; 76 (2): 95-102.

7. Mohammed Al Shamrani. Shahira Al Turkmani. Update Of Intraocular Lens Implantation In Children. Saud Journ of Ophthalmol (2012) 26, 271-275.

8. Alvarado D, Rivera B, Lagos L, Ochoa M, Starkman I, Castillo M, et al. Encuesta Nacional De Ceguera $Y$ Deficiencia Visual Evitables En Honduras. Rev Panam Salud Publica. 2014; 36(5): 300-5.
9. Rojas N, Barahona M, Alvarado D, Alger J. Catarata, Una Causa Prevenible de Ceguera, y Las Líneas Prioritarias de Investigación en Oftalmología En Honduras Rev Med Hondur, Vol. 82, No. 4, 2014.

10. Zimmerman M, Reyes C, Catarata Pediátrica en un País en Vías de Desarrollo: Revisión Retrospectiva de 328 Casos Arq Bras Oftalmol. 74(3): 163-5,2011

11. Castro LP et Al. Resultados del Tratamiento Quirúrgico de la Catarata Traumática en Edad Pediátrica: Rev Cub de Oft; 23(2) 209-218, 2010.

12. Ochoa-Gomez M et Al. Manejo quirúrgico y complicaciones de la catarata congénita. Experiencia de cinco años en la Asociación para Evitar la Ceguera en México.Arch Soc Esp Oftalmol. 2010; 85(1):16-21.

13. Martínez $S$ et al.Cataratas congénitas: complicaciones y resultados funcionales según diferentes técnicas quirúrgicas. Arch Soc Esp Oftalmol. 2010; 85(1): 16-21.

14. Morales M. Cataratas en la Edad Pedíatrica: An Pediatr Contin 2012; 10(2): 65-7. 